Supplement of Saf. Nucl. Waste Disposal, 1, 79-81, 2021

https://doi.org/10.5194/sand-1-79-2021-supplement

(C) Author(s) 2021. CC BY 4.0 License.

Supplement of

\title{
From process to system understanding with multi-disciplinary investigation methods: set-up and first results of the CD-A experiment (Mont Terri rock laboratory)
}

\section{Gesa Ziefle et al.}

Correspondence to: Gesa Ziefle (gesa.ziefle@bgr.de)

The copyright of individual parts of the supplement might differ from the article licence. 
From process to system understanding with multi-disciplinary investigation methods: set-up and first results of the CD-A experiment (Mont Terri Rock Laboratory)

\section{BGR:}

Swisstopo:

Helmholtz (UFZ):

GRS:

ENSI:

KIT/ISU:

BGE:
Gesa Ziefle, Tuanny Cajuhi, Stephan Costabel, Antoine Fourriére, Markus Furche, Jürgen Hesser, Tilo Kneuker, Herbert Kunz, Ben Laurich, Jobst Maßmann, Christian Ostertag-Henning, Dorothee Rebscher Sebastian Condamin, David Jaeggi, Senecio Schefer

Nico Graebling, Olaf Kolditz, Karsten Rink

Larissa Friedenberg, Kyra Jantschik, Oliver Czaikowski, Klaus Wieczorek

Bastian Graupner

Franz Königer, Rainer Schuhmann

Wolfram Rühaak, Marc Wengler 


\section{From process to system understanding} with multi-disciplinary investigation methods: set-up and first results of the CD-A experiment (Mont Terri Rock Laboratory)

\section{Outline}

- Introduction CD-A Experiment

- Process understanding

- System understanding
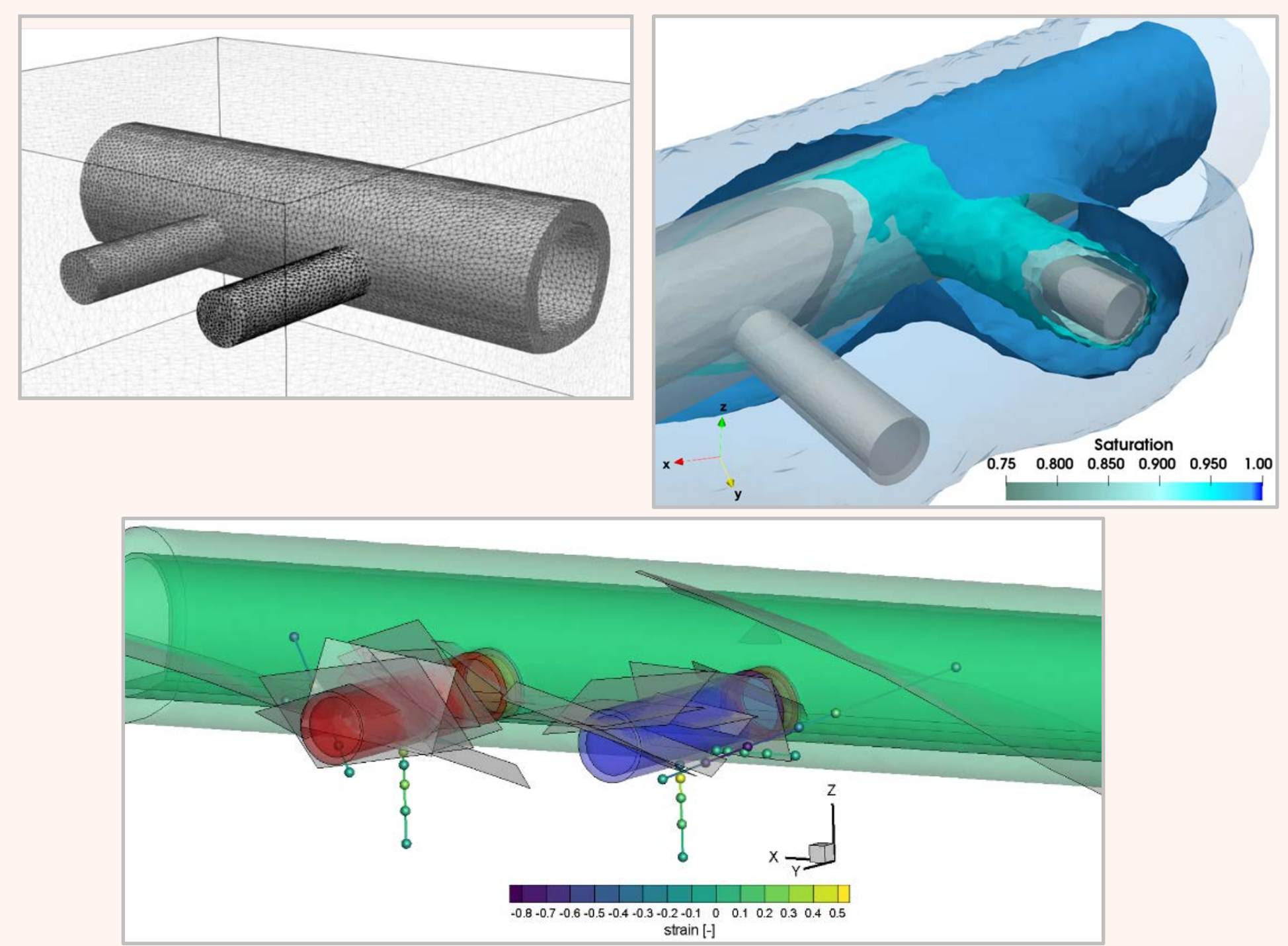


\section{CD-A Experiment: Set-up}
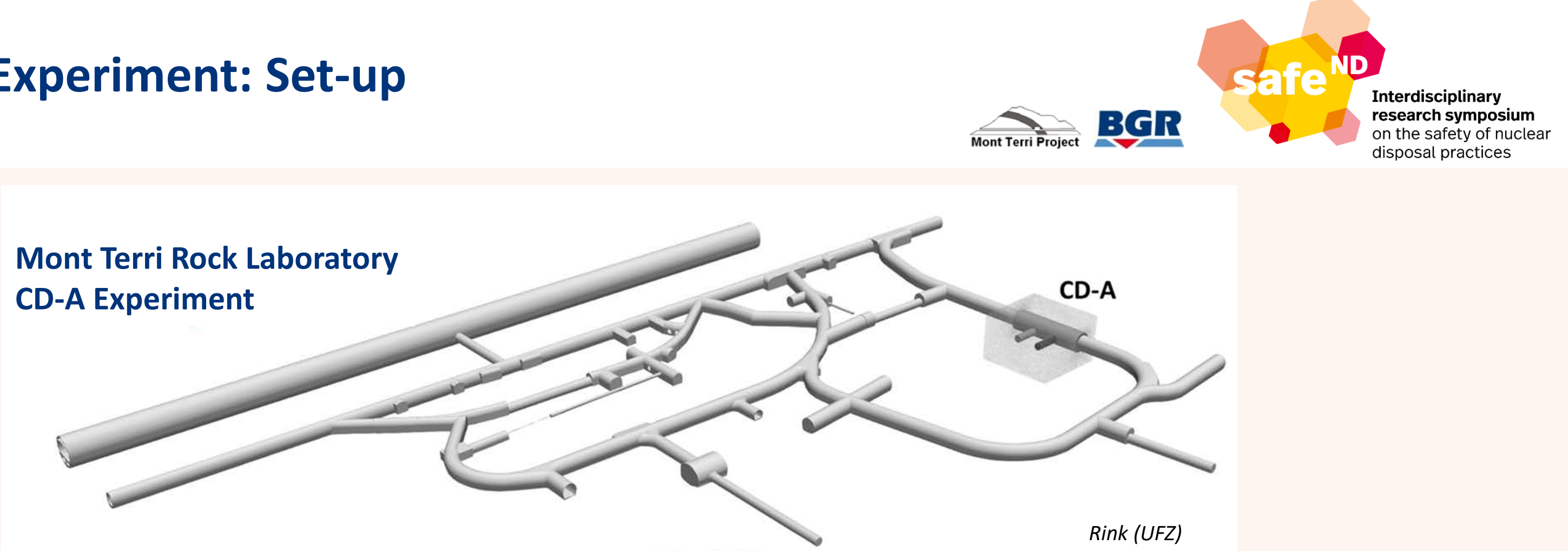

Aim:

Investigation of coupled HM-effects in the Opalinus Clay

Important for:

Stability and integrity of a potential repository

- 2 horizontal excavations

- perpendicular to strike of bedding

- no stabilization

- upper sandy facies

- influence of climatic conditions on HM behavior

- CD: "cyclic deformations"

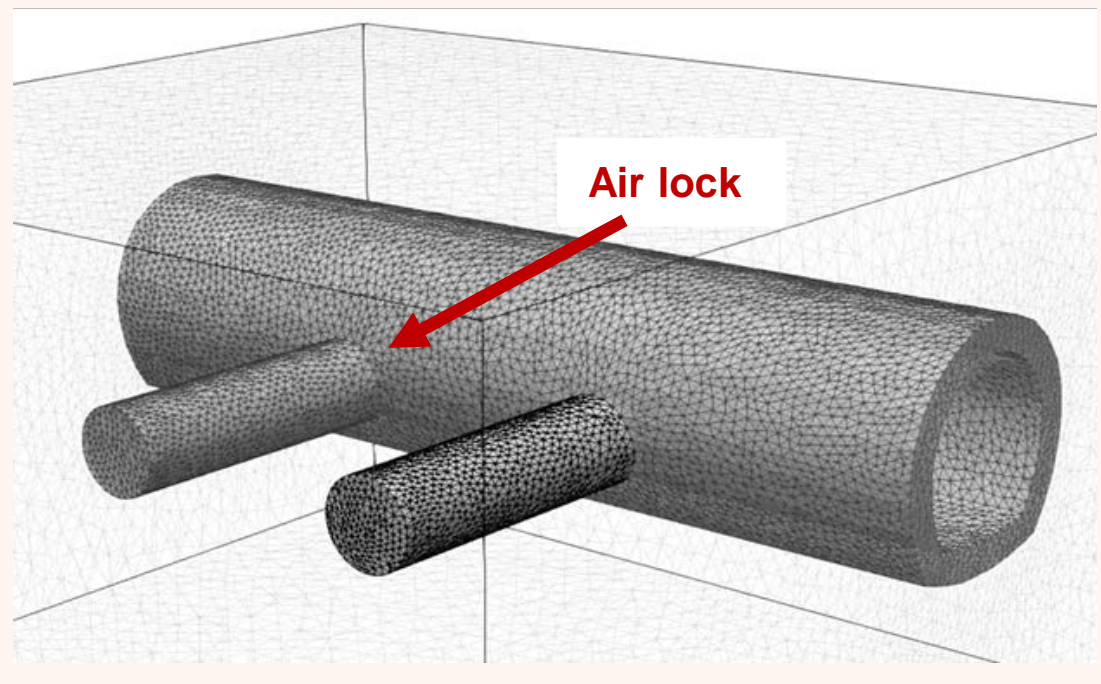




\section{CD-A Experiment: Set-up}

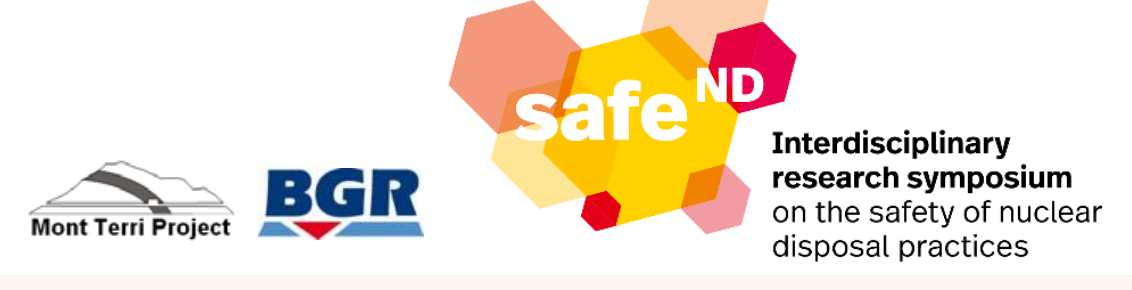

Open twin:

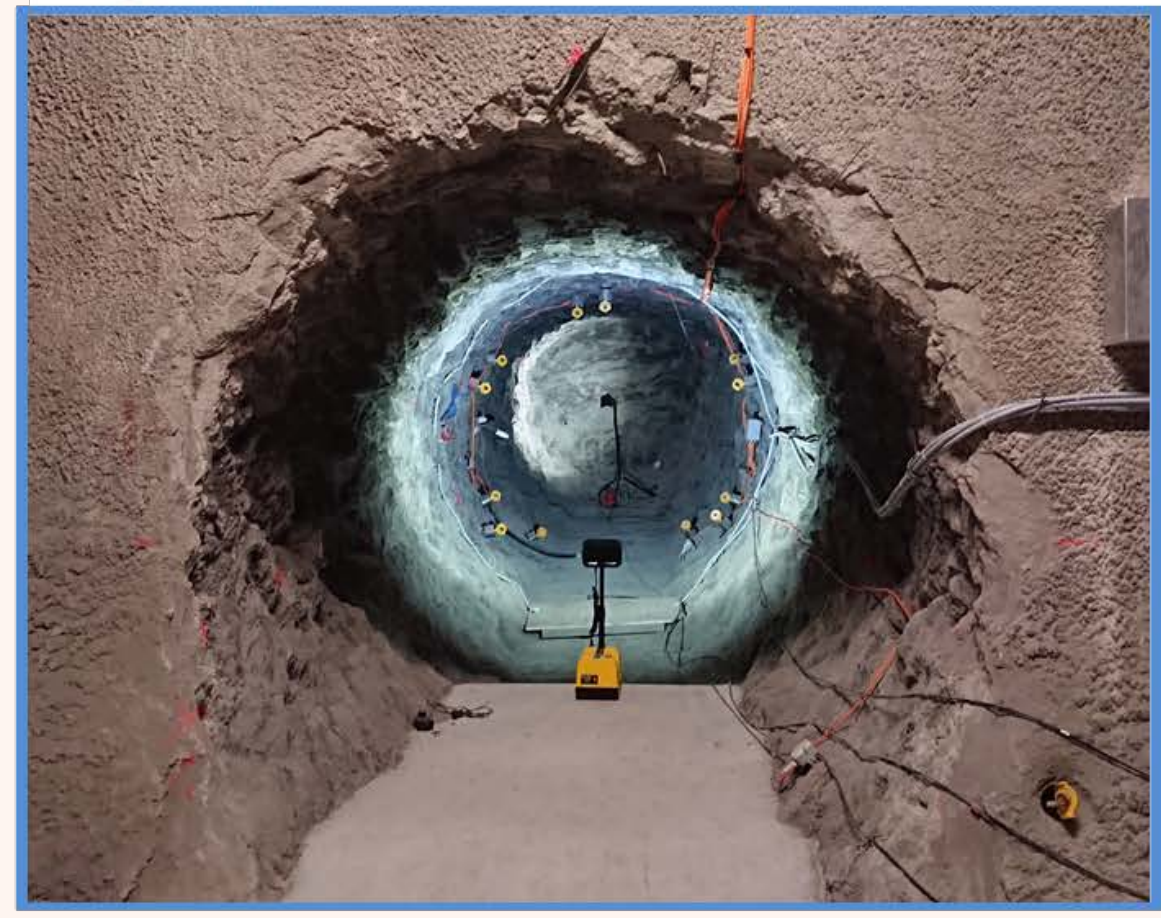

\section{Open twin}

"natural" niche containing coupled $\mathrm{HM}$ effects due to desaturation

\section{Closed twin:}

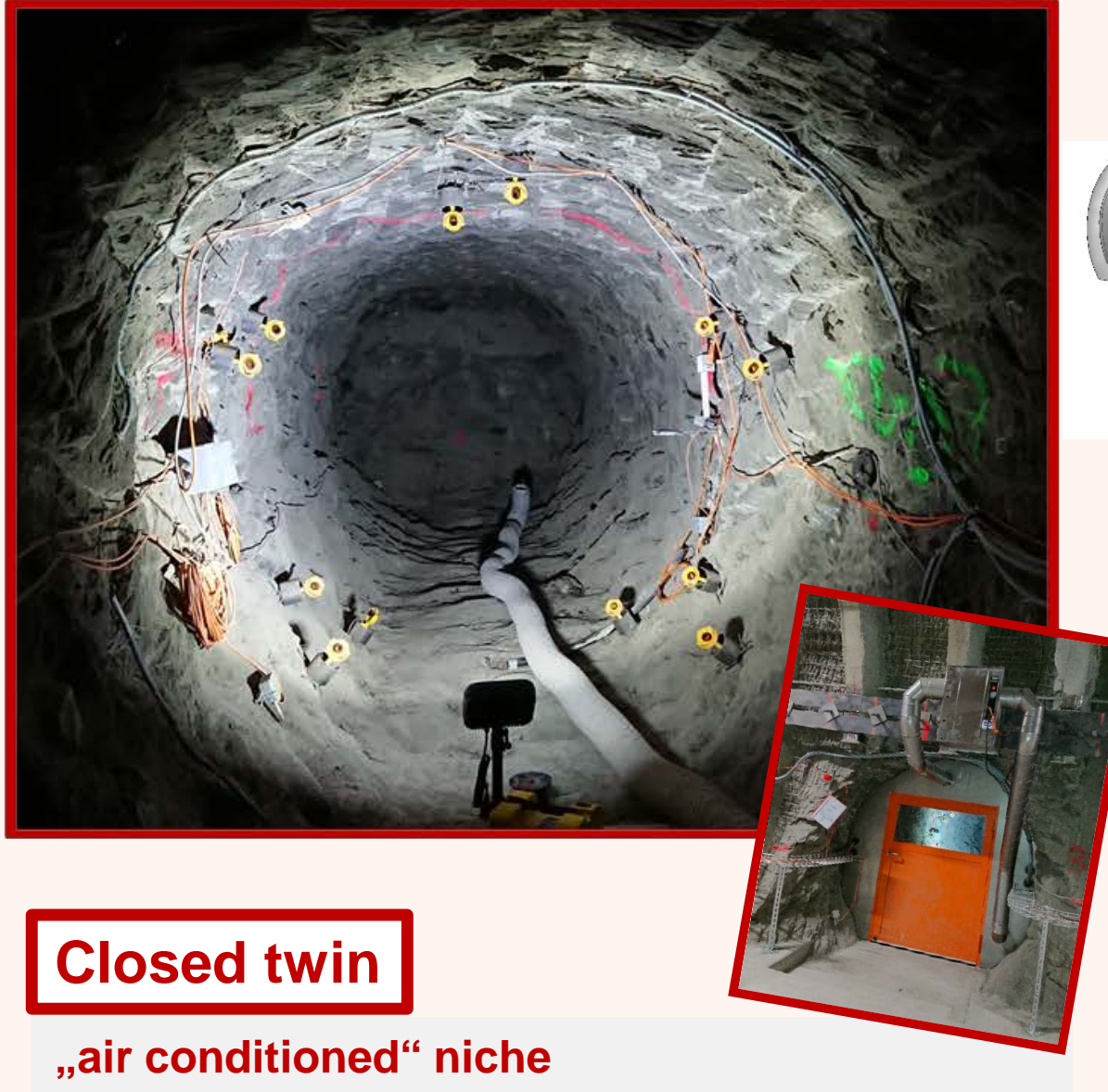

avoiding desaturation effects as much as possible

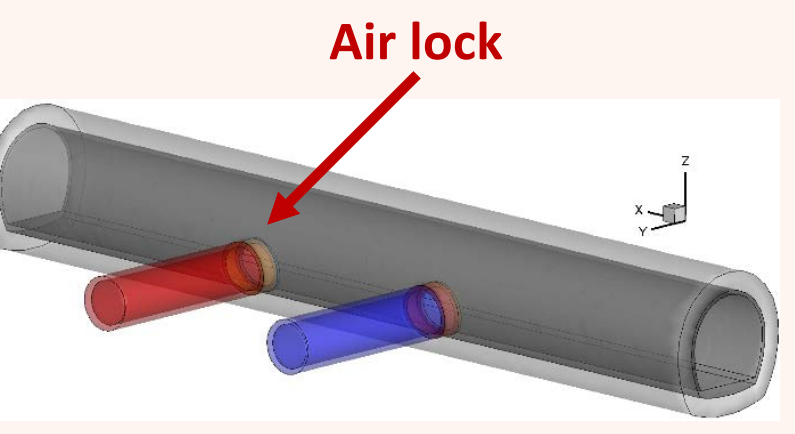




\section{CD-A Experiment: Climatic conditions}
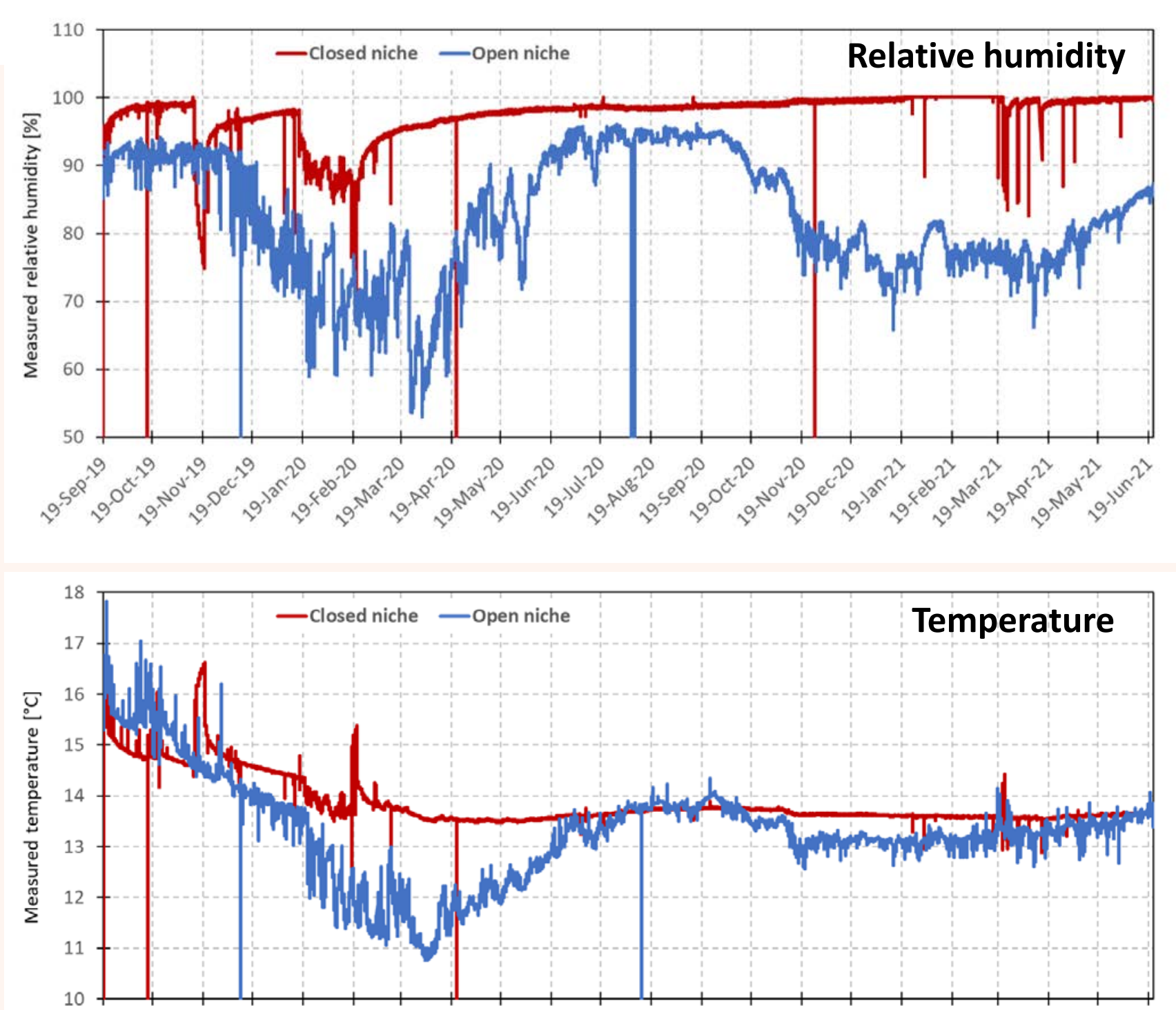

\section{$\overbrace{\text { Mont Terri Project }}^{\text {BCR }}$}

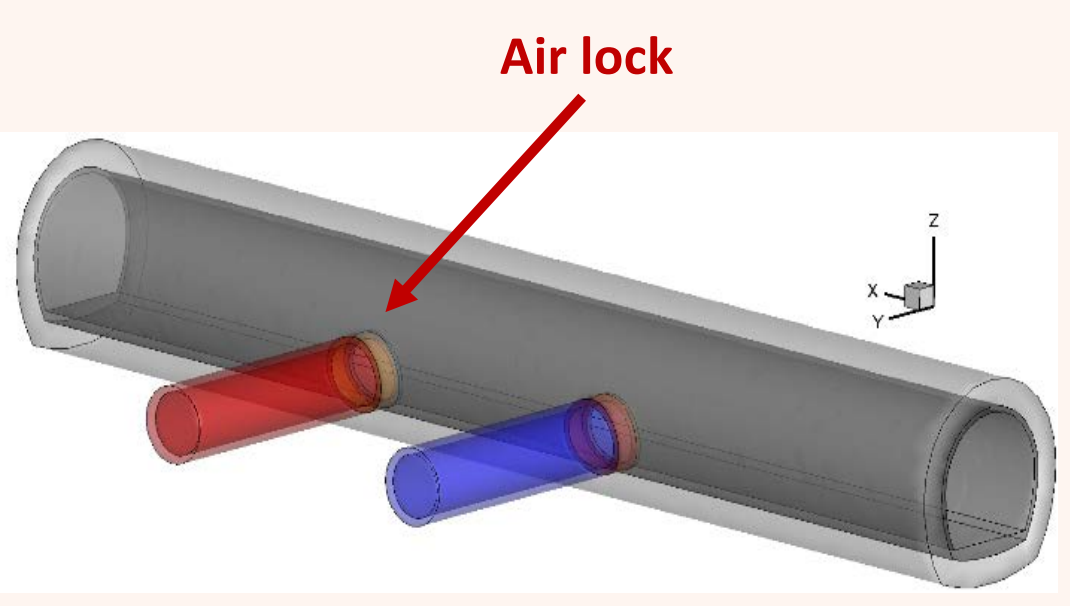

Closed niche:

Constantly high relative humidity

Open niche:

Seasonally influenced relative humidity 


\section{CD-A Experiment: Relevant effects}

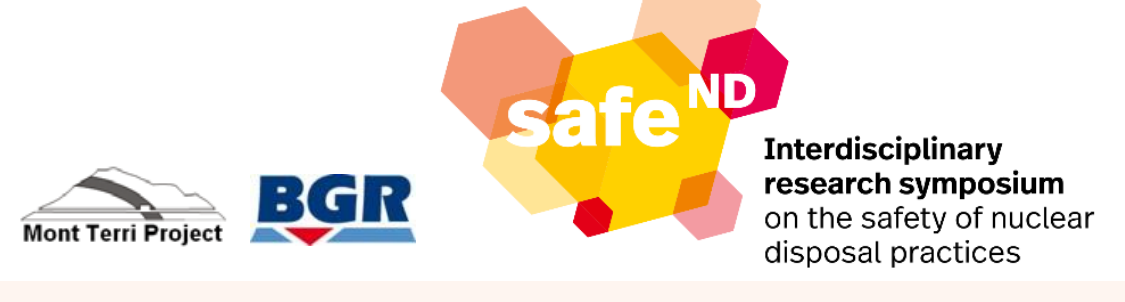

\section{Open twin:}

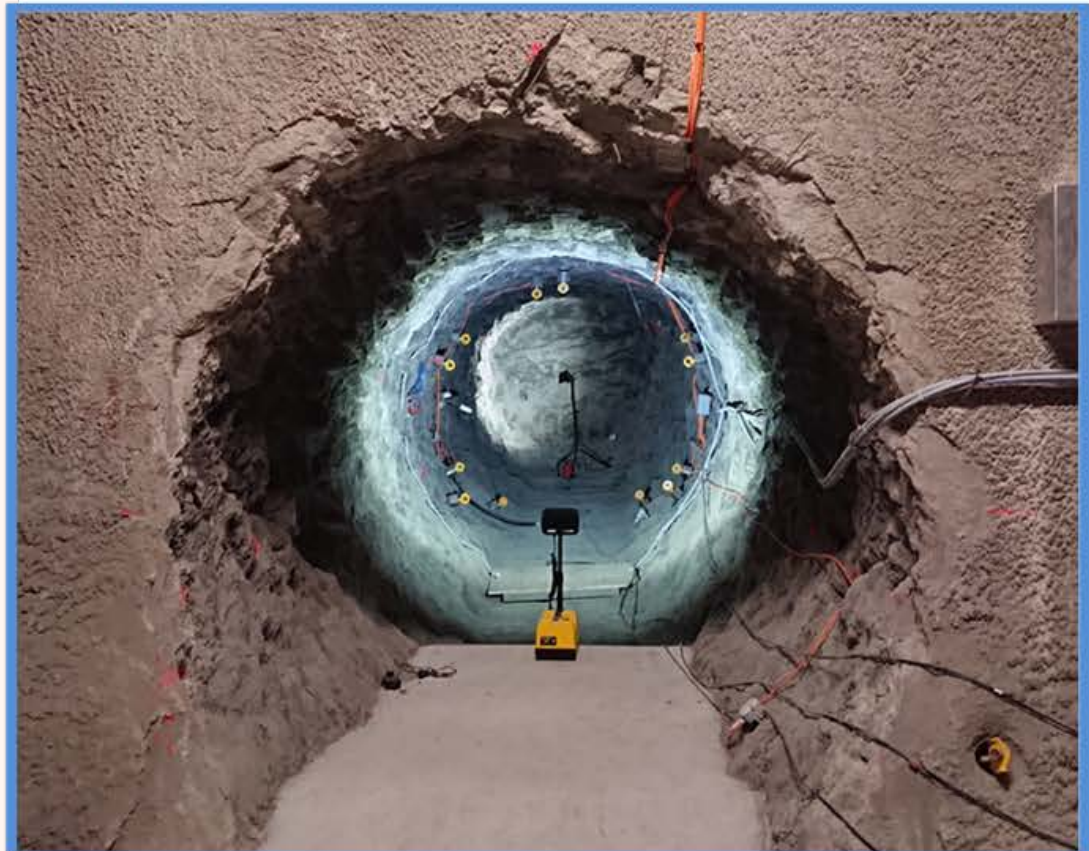

\section{Closed twin:}

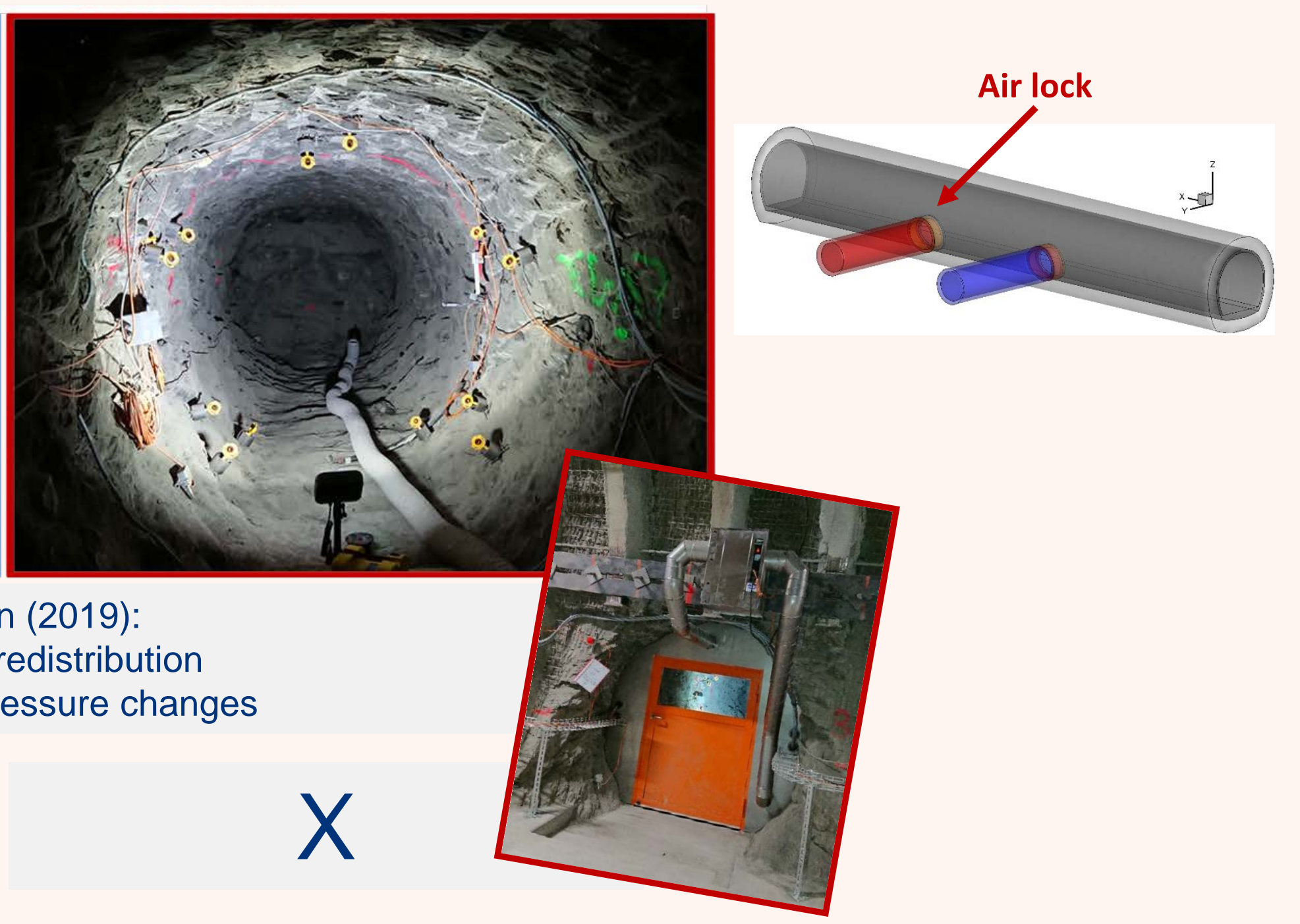

- Climatization

- Desaturation

- Swelling/Shrinkage

- Saturation dependent material parameters
- Excavation (2019)

- Stress redistribution

- Pore pressure changes 


\section{Desaturation around the niches - numerical modelling}

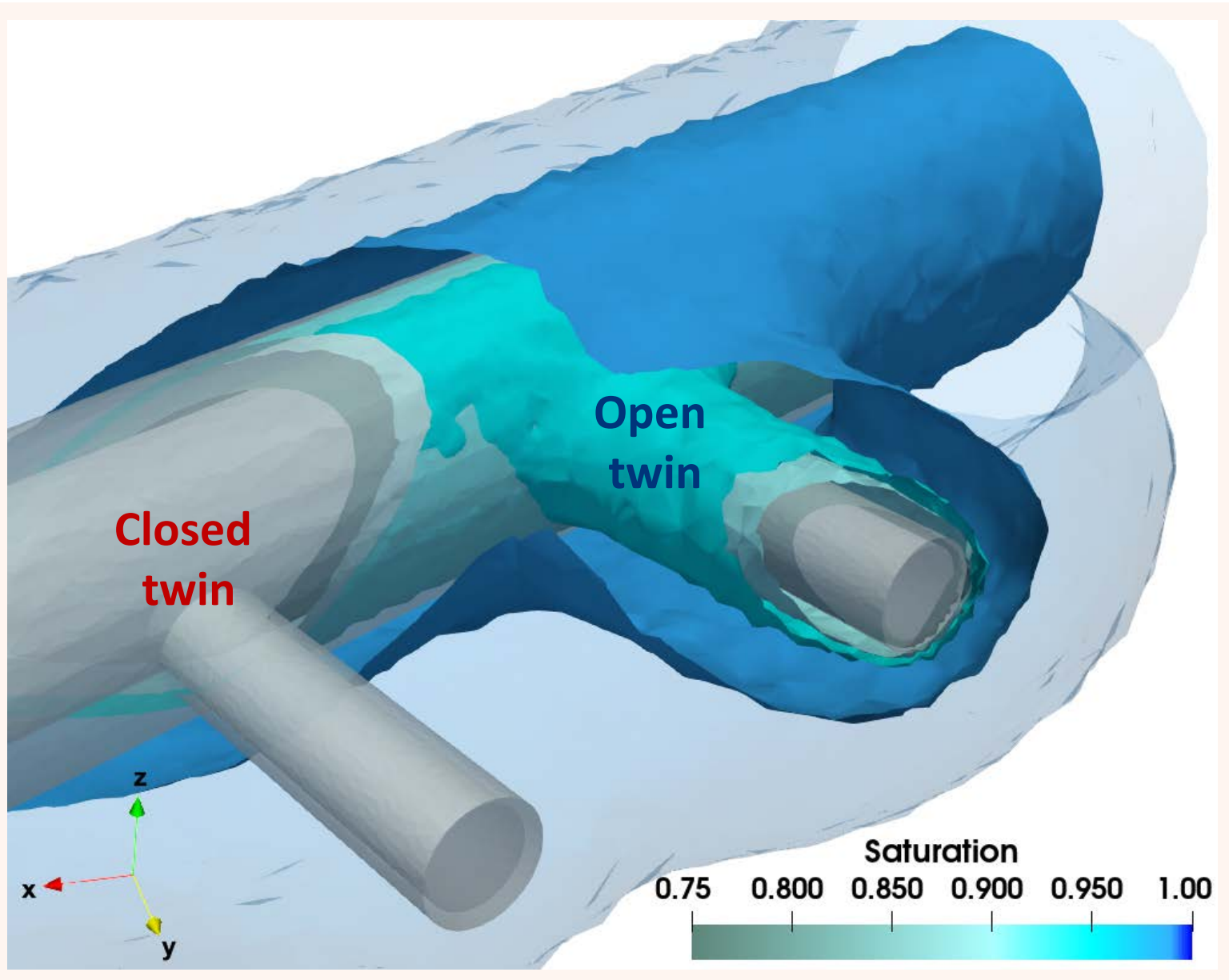

Numerical modelling (OpenGeoSys6) of the twin niches also indicates the differences of the desaturated zone (already) two years after excavation
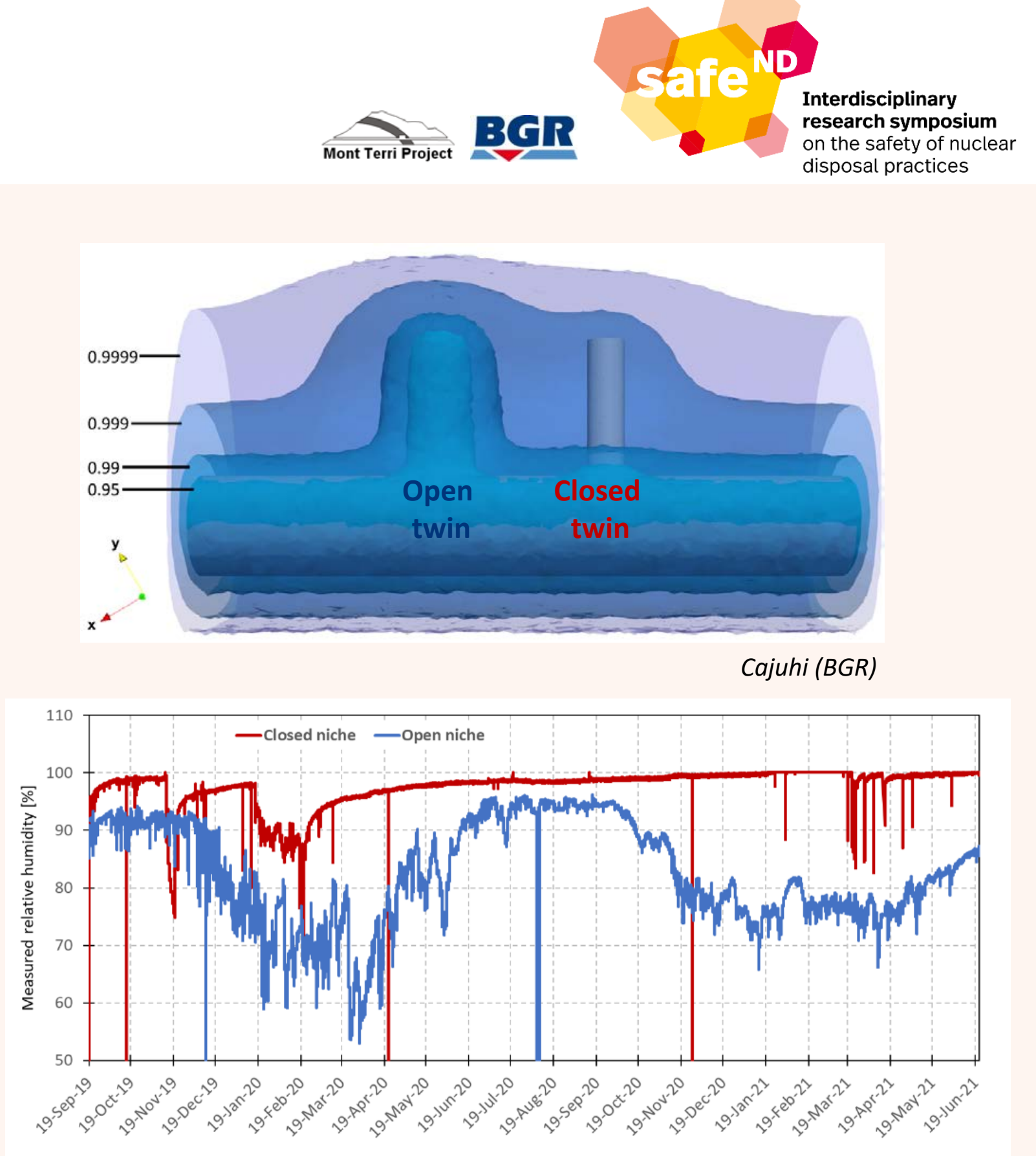


\section{Desaturation around the niches: geophysical measurements}
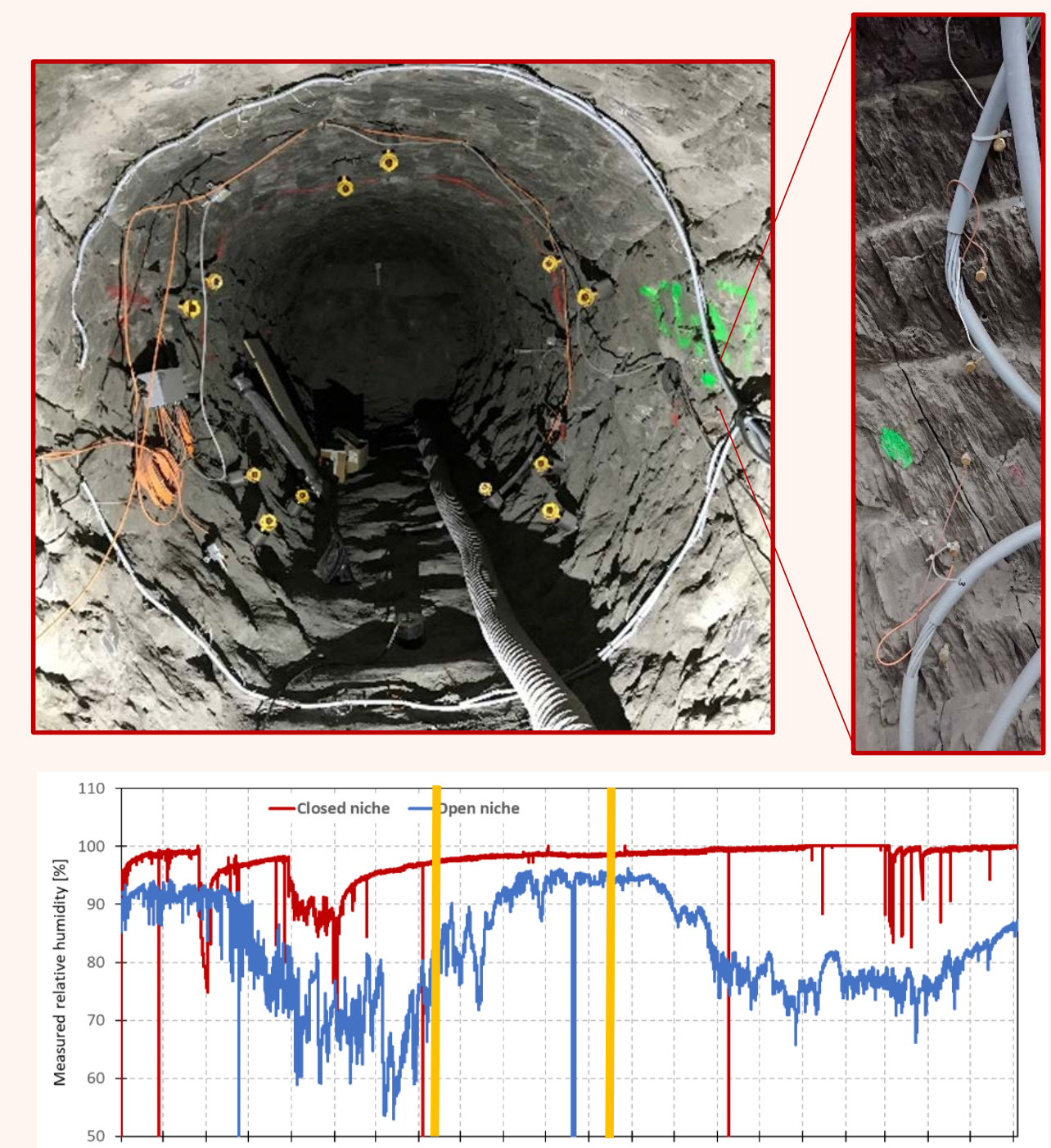

Electric Resistivity Tomography ERT measurements at ring profiles: relative changes of the resistivity

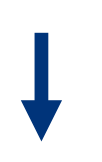

Evolution of desaturated zone

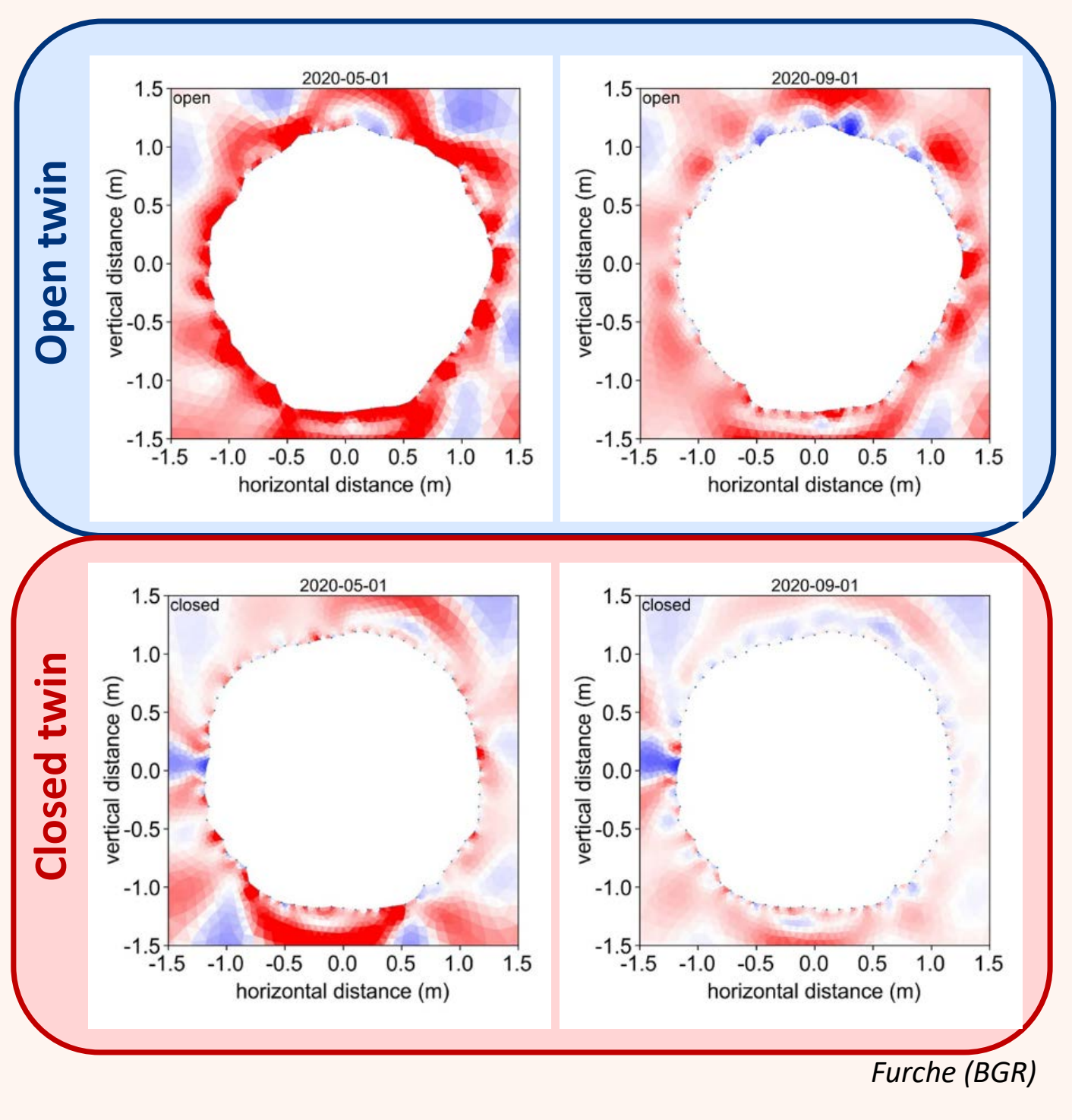




\section{Desaturation around the niches: geophysical measurements}

Nuclear magnetic resonance measurements (NMR) provide information about the water content
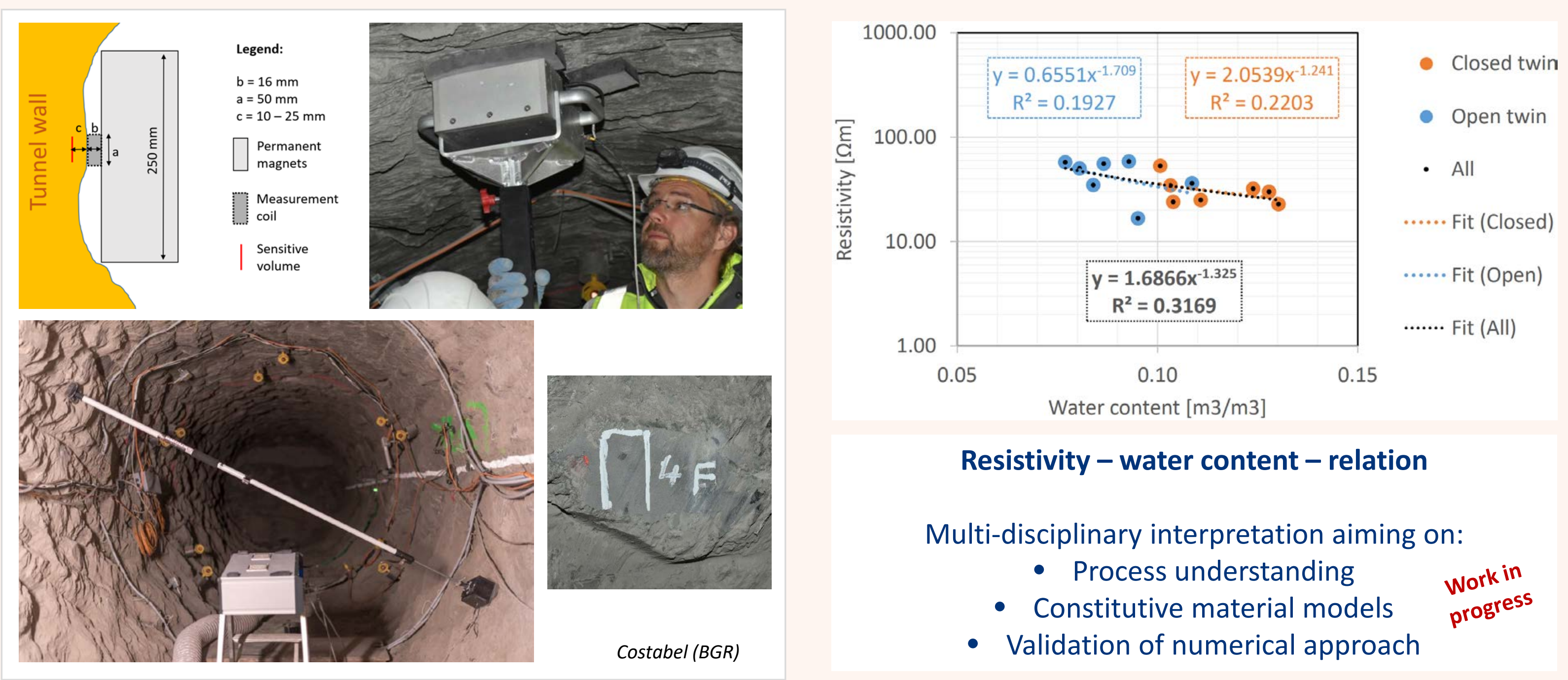

Resistivity - water content - relation

Multi-disciplinary interpretation aiming on:

- Process understanding

- Constitutive material models

- Validation of numerical approach 


\section{Characterization of Opalinus Clay}

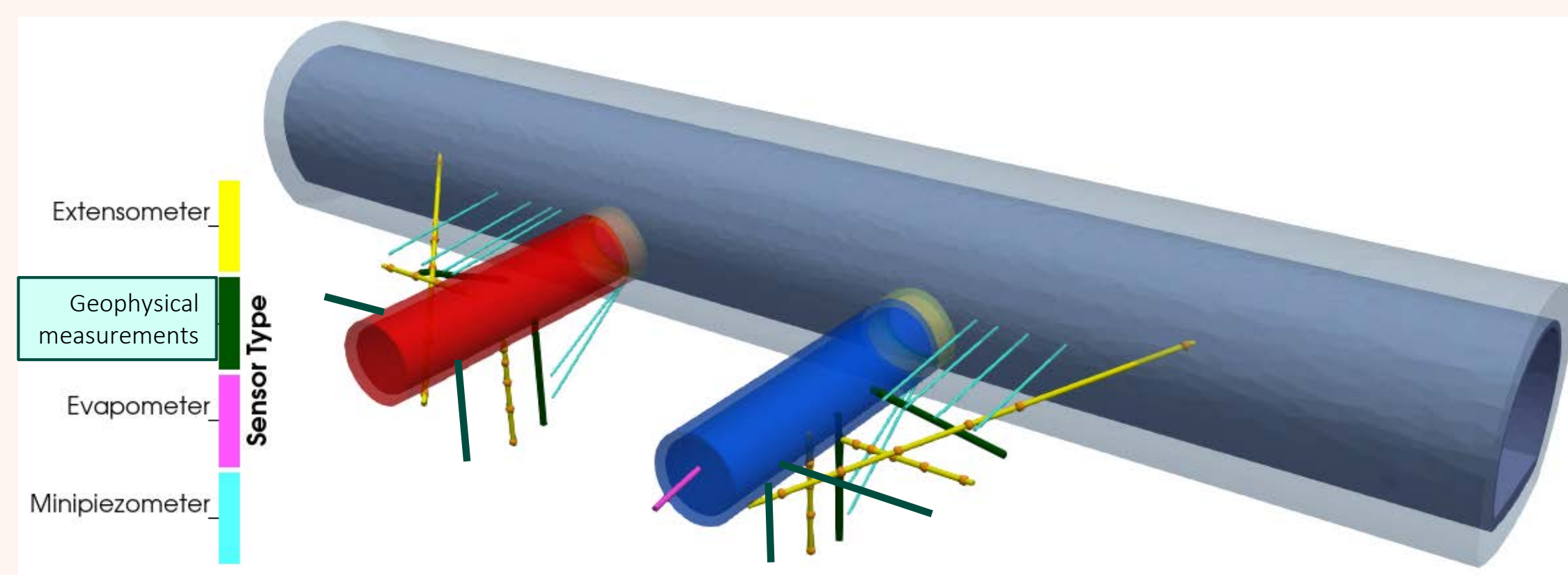

\begin{tabular}{|l|c|c|c|c|c|c|c|}
\hline Mnfo: & Lith. & ERT & MSM & NMR/CCM & Taupe & Pulse test & Numerics \\
\hline Subfacies type & + & + & - & - & - & - & $\left(^{*}\right)$ \\
\hline EDZ & - & - & + & - & + & $(+)$ & $\left(^{*}\right)$ \\
\hline Fault zones & + & - & - & - & $(+)$ & $(+)$ & $\left(^{*}\right)$ \\
\hline Water content & - & + & - & + & $(+)$ & - & + \\
\hline
\end{tabular}

Table 2: Investigation methods and available information. [ +: information available, -: no information available, *: may provide information about their impact on physical effects, (): limited information]. Lith. = Lithological investigations on drill cores.
ERT:

Electric Resistivity tomography

MSM:

Mini-Seismic

NMR:

Nuclear Magnetic Resonance

CCM:

Calciumcarbid-Method

Taupe:

Time domain reflectometry (TDR) 


\section{Borehole investigations}
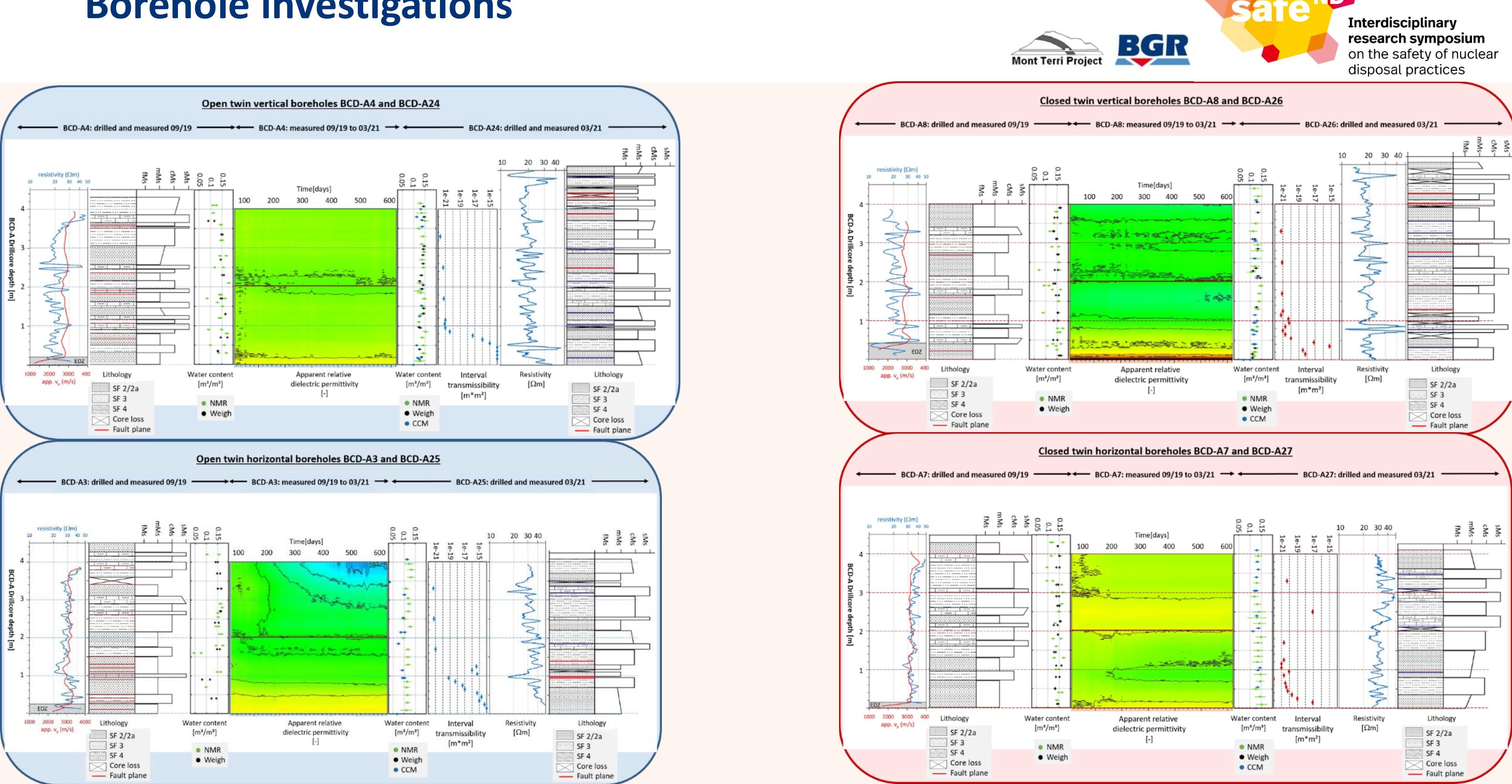


\section{Borehole investigations}

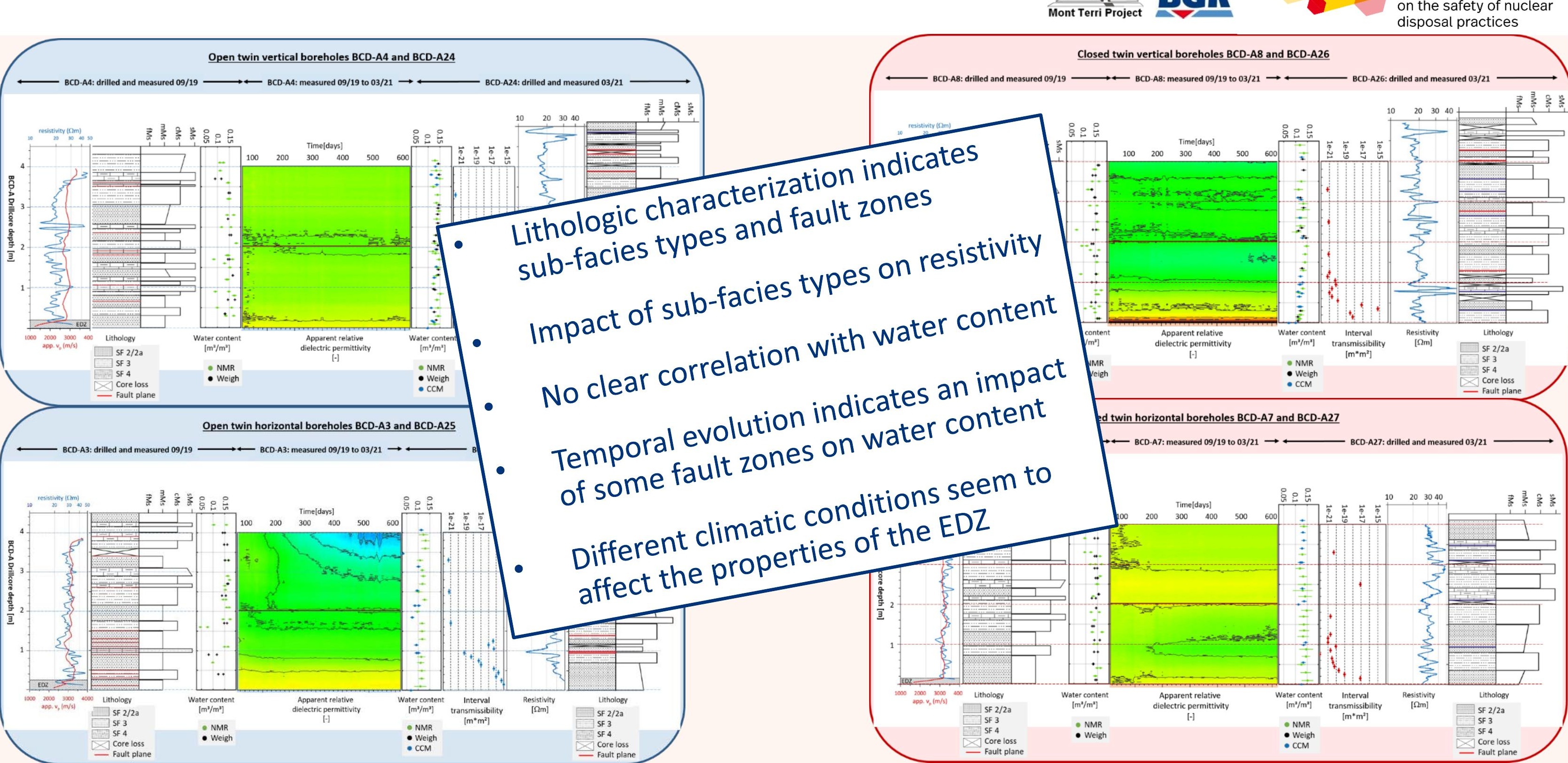




\section{Extensometer measurements in relation to detected fault zones}

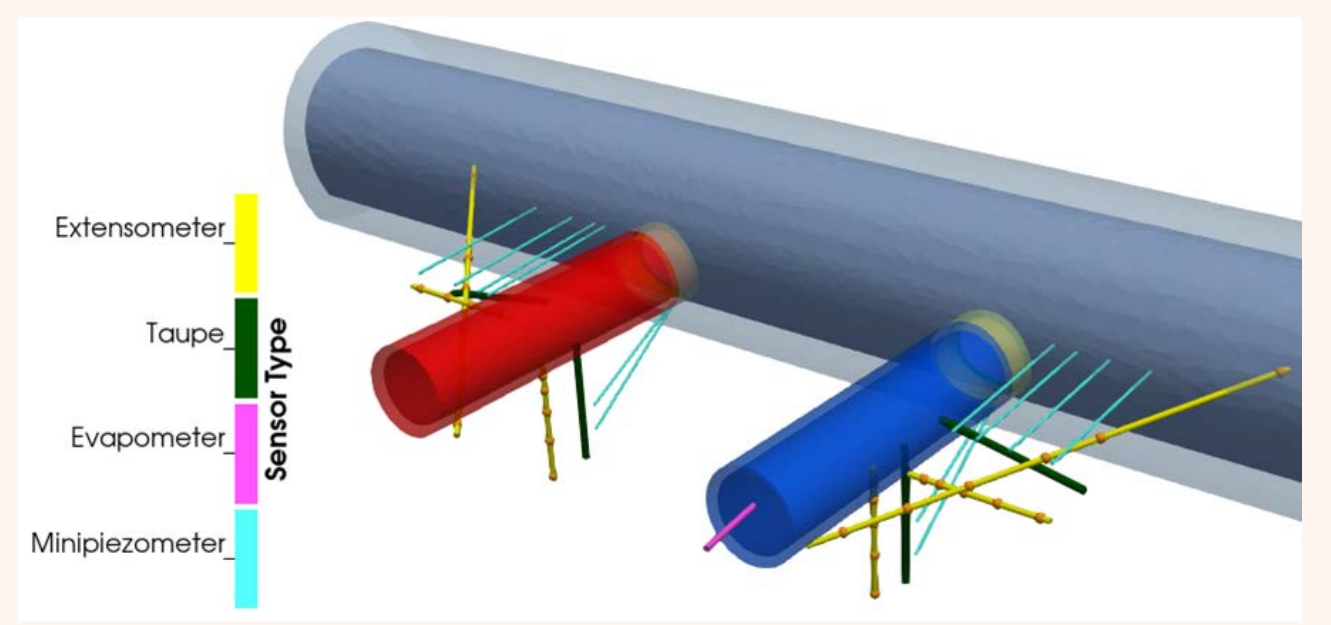

Measurements indicate impact of some fault zones on convergence behavior

Further investigations needed!
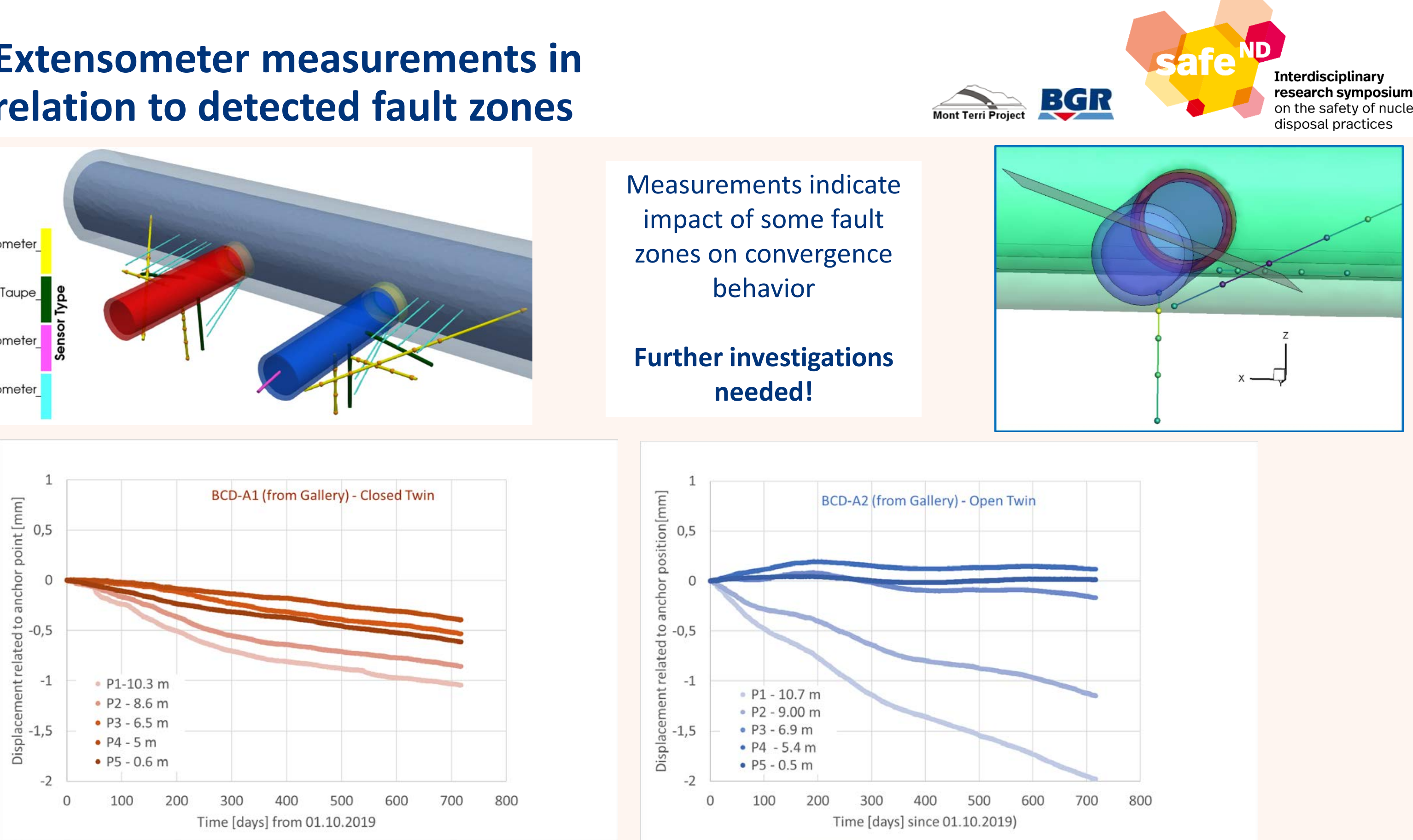


\section{Conclusion}

\section{CD-A Experiment:}

- Increased process understanding of coupled long-term HM effects

- Geologic characterization + geophysical measurements + numerical modelling + visualization

- Multi-disciplinary approach + close collaboration

$$
\begin{aligned}
& \text { Thanks to the } \\
& \text { CD-A Team!!! }
\end{aligned}
$$

- Aiming on an increased system understanding

- Calibration/Verification of constitutive modelling approaches

\section{Preliminary results:}

- Measurements and numerical modelling illustrate desaturation and convergence around the twin niches

- Impact of heterogeneities such as sub-facies types and fault zones on coupled HM effects 